\title{
Penalty-based Heuristic DIRECT Method for Constrained Global Optimization
}

\author{
M. Fernanda P. Costa $(\varangle)^{1}$, \\ Ana Maria A. C. Rocha ${ }^{2}$, and Edite M. G. P. Fernandes ${ }^{2}$ \\ 1 Centre of Mathematics, \\ University of Minho, 4710-057 Braga, Portugal \\ mf c@math. uminho.pt \\ 2 ALGORITMI Center, \\ University of Minho, 4710-057 Braga, Portugal, \\ \{arocha, emgpf\}@dps.uminho.pt
}

\begin{abstract}
This paper is concerned with an extension of the heuristic DIRECT method, presented in 8], to solve nonlinear constrained global optimization (CGO) problems. Using a penalty strategy based on a penalty auxiliary function, the CGO problem is transformed into a bound constrained problem. We have analyzed the performance of the proposed algorithm using fixed values of the penalty parameter, and we may conclude that the algorithm competes favourably with other DIRECT-type algorithms in the literature.
\end{abstract}

Keywords: Global optimization - DIRECT method · Heuristic · Penalty auxiliary function

\section{Introduction}

In this paper, we aim to find the global solution of a non-smooth and non-convex constrained optimization problem using a non-differentiable penalty function and the DIRECT method [1. The constrained global optimization (CGO) problem has the form:

$$
\begin{aligned}
\min _{x \in \Omega} & f(x) \\
\text { subject to } & h(x)=0 \\
& g(x) \leq 0,
\end{aligned}
$$

where $f: \mathbb{R}^{n} \rightarrow \mathbb{R}, h: \mathbb{R}^{n} \rightarrow \mathbb{R}^{m}$ and $g: \mathbb{R}^{n} \rightarrow \mathbb{R}^{p}$ are nonlinear continuous functions and $\Omega=\left\{x \in \mathbb{R}^{n}:-\infty<l_{i} \leq x_{i} \leq u_{i}<\infty, i=1, \ldots, n\right\}$. Denoting the feasible set of problem (1) by $\mathcal{F}=\{x \in \Omega: h(x)=0, g(x) \leq 0\}$, we define a non-negative function

$$
\theta(x)=\sum_{i=1}^{m}\left|h_{j}(x)\right|+\sum_{i=1}^{p} \max \left\{g_{i}(x), 0\right\},
$$

where $\theta(x)=0$ if $x \in \mathcal{F}$. Since convexity is not assumed, many local minima may exist in the feasible region, although we require only a global solution. For 
non-smooth problems, the derivative-free methods are the most appropriate. Deterministic and stochastic methods have been proposed to solve CGO problems [2]3. Using deterministic methods, the convergence to a global optimal solution can be guaranteed and a solution with a required accuracy is obtained in a finite number of steps. On the other hand, stochastic methods are not guaranteed to find a global optimal solution although they are often able to find very good solutions after a (moderate) large number of steps. Stochastic convergence may be established using probability theory.

From the class of deterministic methods, the DIRECT method [1] has proven to be quite effective in converging to the global solution while avoiding to be trapped in a local solution, as far as bound constrained global optimization problems are concerned. The method has attracted considerable interest from the research community and several strategies have been incorporated into DIRECT, including the local search reinforcement 445, the improvement of the global search [6], new ideas for the selection of potentially optimal hyperrectangles [78, and new partition schemes 91011.

The most popular methods to solve the problem (1) combine the objective function with a penalty term that aims to penalize constraint violation. Penalty functions within a DIRECT-type framework are proposed in [12 13/14. An auxiliary function that combines in a special manner information on the objective and constraints is presented in [15]. Other techniques that involve the handling of the objective function and constraints violation separately can be found in 5[16 17.

The main contribution of this paper is the following. The two-phase heuristic DIRECT algorithm, proposed by the authors in [8], is extended to solve CGO problems using an auxiliary penalty function. The auxiliary function proposed in [15] is redefined to transform the CGO problem (1) into a bound constrained global optimization (BCGO) problem.

The paper is organized as follows. Section 2 briefly presents some ideas and the main steps of the DIRECT method. Section 3 describes a heuristic incorporated into the DIRECT algorithm to reduce the number of identified potentially optimal hyperrectangle, and the corresponding proposed extension to handle CGO problems, in particular, the use of a non-differentiable auxiliary penalty function. Finally, Sect. 4 contains the results of our preliminary numerical experiments and we conclude the paper with the Sect. 5 .

\section{DIRECT Method}

The DIRECT (DIviding RECTangles) algorithm [1], originally proposed to solve BCGO problems of the form

$$
\min _{x \in \Omega} f(x),
$$

assumes that the objective function $f$ is a continuous function, and iteratively produces finer and finer partitions of the hyperrectangles generated from $\Omega$ (see also [18]). The algorithm is a modification of the standard Lipschitzian approach, 
where $f$ is assumed to satisfy the Lipschitz condition,

$$
\left|f\left(x_{1}\right)-f\left(x_{2}\right)\right| \leq K\left\|x_{1}-x_{2}\right\| \text { for all } x_{1}, x_{2} \in \Omega,
$$

and the Lipschitz constant $K>0$ is viewed as a weighting parameter that indicates how much emphasis to place on global versus local search. DIRECT is a deterministic method that does not require any analytical or numerical derivative information and searches (locally and globally) the feasible region $\Omega$ for hyperrectangles that are known as potentially optimal hyperrectangle (POH). These POH satisfy the two conditions established in the following definition:

Definition 1. Given the partition $\left\{\mathcal{H}^{i}: i \in H\right\}$ of $\Omega$, let $\epsilon$ be a positive constant, and let $f_{\min }$ be the current best function value. A hyperrectangle $j$ is said to be potentially optimal if there exists some rate-of-change constant $\hat{K}^{j}>0$ such that

$$
\begin{aligned}
& f\left(c_{j}\right)-\frac{\hat{K}^{j}}{2}\left\|u^{j}-l^{j}\right\| \leq f\left(c_{i}\right)-\frac{\hat{K}^{i}}{2}\left\|u^{i}-l^{i}\right\|, \text { for all } i \in H \\
& f\left(c_{j}\right)-\frac{\hat{K}^{j}}{2}\left\|u^{j}-l^{j}\right\| \leq f_{\min }-\epsilon\left|f_{\min }\right|
\end{aligned}
$$

where $c_{j}$ (resp. $c_{i}$ ) is the center and $\left\|u^{j}-l^{j}\right\| / 2$ (resp. $\left\|u^{i}-l^{i}\right\| / 2$ ) represents the size of hyperrectangle $j \in H$ (resp. $i$ ), and $H$ is the set of indices of the hyperrectangles at the current iteration [115].

The use of $\hat{K}^{j}$ in the definition intends to show that it is not the Lipschitz constant. The second condition in (4) aims to prevent the algorithm from identifying as $\mathrm{POH}$ the hyperrectangle with center that corresponds to $f_{\min }$. This way, small hyperrectangles where very small improvements may be obtained are skipped to be further divided.

The most important step in the DIRECT algorithm is the identification of $\mathrm{POH}$ since it determines the search along the feasible set. Each identified hyperrectangle is trisected along its longest sides and two new points in the hyperrectangle are sampled and remain center points of the other hyperrectangles (of the trisection).

A global search driven strategy would identify POH from the biggest hyperrectangles. On the other hand, a local search driven strategy would identify $\mathrm{POH}$ whose center point corresponds to $f_{\min }$. Good solutions are found rather quick but the hyperrectangle that contains the global solution may be missed if its center point has a bad function value. The main steps of the DIRECT algorithm are shown in Algorithm 1 . 


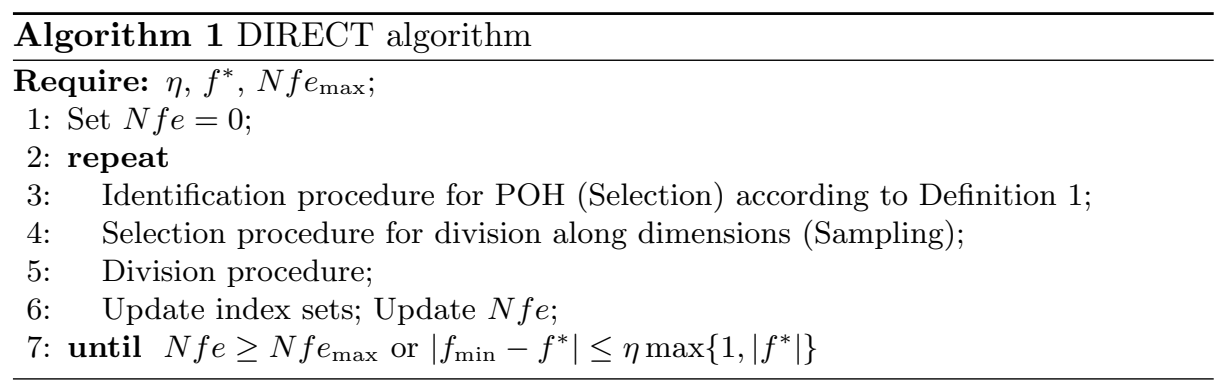

\section{Heuristic DIRECT Method Based on Penalties}

This section presents the extension of a heuristic DIRECT algorithm 8 to handle nonlinear equality and inequality constraints.

\subsection{Heuristic DIRECT Method}

Firstly, we briefly describe a heuristic that can be incorporated into the DIRECT algorithm [8] aiming

- to divide a promising search region into three subregions, so that the number of hyperrectangles that are candidate to be potentially optimal is reduced;

- to choose between a global search driven phase or a local search driven phase.

Since avoiding the identification of $\mathrm{POH}$ that were mostly divided can enhance the global search capabilities of DIRECT [ [ ] and identifying POH that are close to the hyperrectangle which corresponds to $f_{\min }$ may improve the local search process, the heuristic incorporated into the DIRECT method divides the region of the hyperrectangles with least function values in each size group - denoted by candidate hyperrectangles - into three subregions.

Each subregion is defined by the indices based on size of the hyperrectangles. The larger the size the smaller the index. The leftmost subregion includes hyperrectangles whose indices are larger than $i_{l}=\left\lfloor 2 / 3 i_{\min }\right\rfloor$, where $i_{\min }$ is the index of the hyperrectangle that corresponds to $f_{\min }$. The rightmost subregion contains the hyperrectangles with indices that are smaller than $i_{u}=\left\lfloor 1 / 3 i_{\min }\right\rfloor$ and the middle subregion contains hyperrectangles with indices between $i_{l}$ and $i_{u}$ (including these limits).

To be able to guarantee convergence to the global solution while avoiding the stagnation in a local solution, the algorithm cycles between global and local search phases. It starts with a global driven search, where $G_{\max }$ iterations are performed using all candidate hyperrectangles from the rightmost subregion, $50 \%$ of the candidate hyperrectangles from the middle subregion (randomly selected) and $10 \%$ of the candidate hyperrectangles from the leftmost subregion (randomly selected). This choice of percentages is hereinafter denoted by $(10 / 50 / 100) \%$. At each iteration, the set of $\mathrm{POH}$ are identified among these selected hyperrectangles. Afterwards, a local driven search is implemented for $L_{\max }$ iterations 
with the percentages of selected candidate hyperrectangles in the leftmost and rightmost subregions changed, denoted by $(100 / 50 / 10) \%$. This cycling process is repeated until convergence.

\subsection{Penalty Auxiliary Function}

We now extend this heuristic DIRECT method to handle nonlinear equality and inequality constraints. We use an auxiliary function that takes into consideration the violation of inequality constraints by combining information of the objective and constraint functions [15. This function penalizes any deviation of the function value at the center $c_{j}$ of a hyperrectangle above the global optimal value $f^{*}$ :

$$
P\left(c_{j}\right)=\max \left\{f\left(c_{j}\right)-f^{*}, 0\right\}+\sum_{i=1}^{p} \mu_{i} \max \left\{g_{i}\left(c_{j}\right), 0\right\}
$$

where $\mu_{i}$ are positive weighting coefficients. Note that when the hyperrectangle has a feasible center point $c_{j}$, the second term is zero, and when it is infeasible, the second term is positive and the first term only counts for the cases where $f\left(c_{j}\right)$ is above $f^{*}$. Since $f^{*}$ is unknown in general, but satisfies $f^{*} \leq f_{\min }-\varepsilon$, for a small tolerance $\varepsilon>0$, we redefine the following variant of the auxiliary function

$$
P(x ; \mu)=\max \left\{f(x)-\left(f_{\min }-\varepsilon\right), 0\right\}+\mu\left(\sum_{i=1}^{m}\left|h_{i}(x)\right|+\sum_{i=1}^{p} \max \left\{g_{i}(x), 0\right\}\right)
$$

where $f_{\min }$ is the current best function value found so far among all feasible center points. Although different weights might prove to be useful for some problems, we consider only one constant weighting coefficient for all the constraints, and extend the penalized constraint violation term to the equality constraints, since in our formulation they are treated separately from the inequality constraints. We remark that if no feasible point has been found so far, the function $P(x ; \mu)$ is reduced to the second term alone in (6).

The definition of $\mathrm{POH}$ (recall Definition 1 above) is now adapted to the strategy that aims to find a global minimum solution of the problem

$$
\min _{x \in \Omega} P(x ; \mu)
$$

for a fixed $\mu>0$ value, in the sense that the sequence of approximations $x_{\min }^{k}$ (resp. $f_{\min }^{k}$ ) converges to $x^{*}\left(\operatorname{resp} . f^{*}\right)$, the global optimal solution of problem (1), as $k$ increases. In this context, the new algorithm searches (locally and globally) the feasible region $\Omega$ to identify hyperrectangles that are known as POH with respect to $P(x ; \mu)$ and satisfy:

Definition 2. Given the partition $\left\{\mathcal{H}^{i}: i \in H\right\}$ of $\Omega$, let $\epsilon>0$ and $\mu>0$ be constants and let $f_{\min }$ be the current best function value among feasible center 
points. A hyperrectangle $j$ is said to be potentially optimal with respect to $P(x ; \mu)$ if there exists some rate-of-change constant $\hat{K}^{j}>0$ such that

$$
\begin{aligned}
& P\left(c_{j} ; \mu\right)-\frac{\hat{K}^{j}}{2}\left\|u^{j}-l^{j}\right\| \leq P\left(c_{i} ; \mu\right)-\frac{\hat{K}^{i}}{2}\left\|u^{i}-l^{i}\right\|, \text { for all } i \in H \\
& P\left(c_{j} ; \mu\right)-\frac{K^{j}}{2}\left\|u^{j}-l^{j}\right\| \leq P_{\min }-\epsilon\left|P_{\min }\right|
\end{aligned}
$$

where $P_{\min }$ is the current best penalty function value and $H$ is the set of indices of the selected candidate hyperrectangles at the current iteration.

The main steps of the proposed penalty-based heuristic DIRECT algorithm are presented in Algorithm 2.

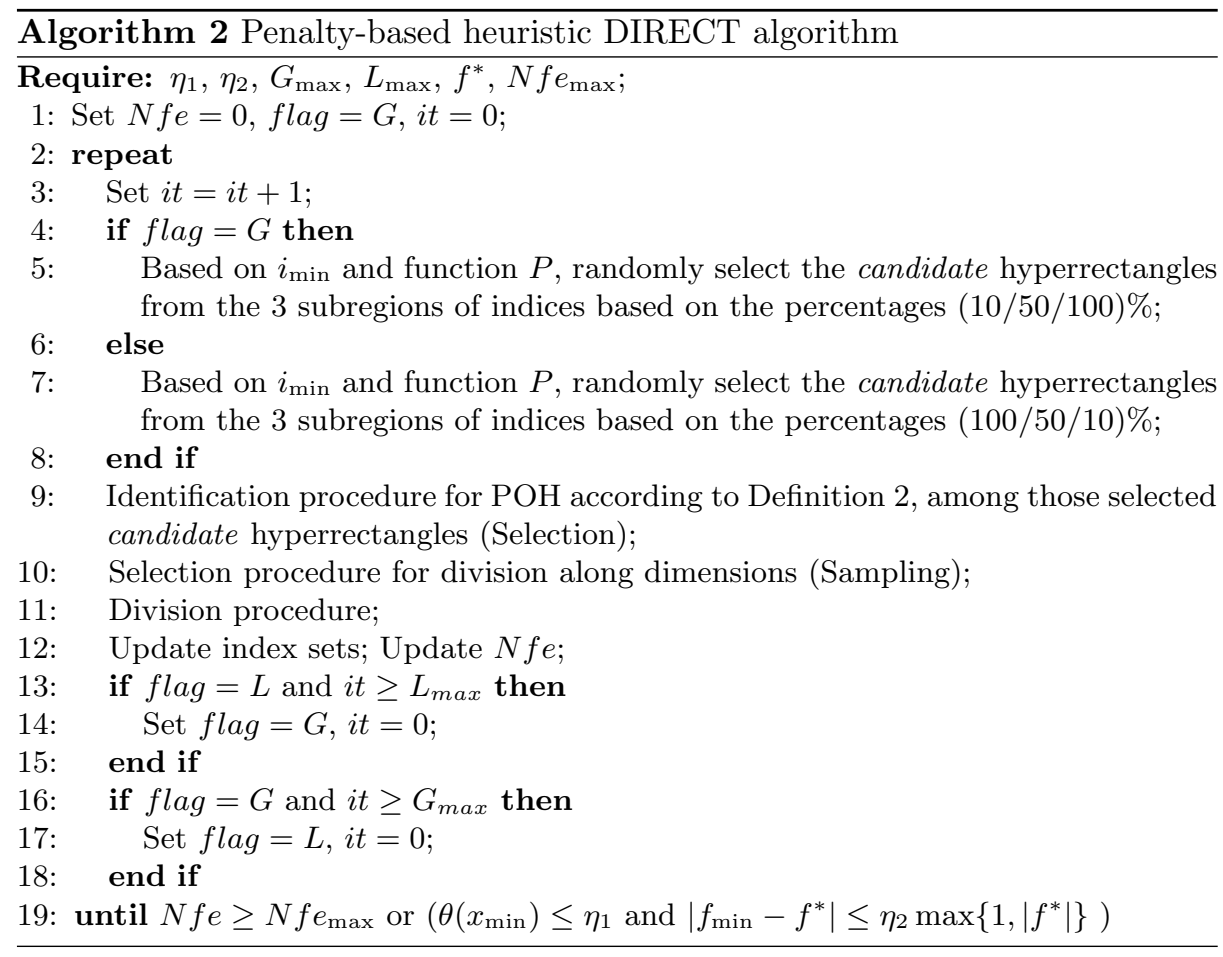

Unless otherwise stated, the stopping conditions for the algorithm are the following. We consider that a good approximate solution $x^{k}$, at iteration $k$, is found, if the conditions

$$
\theta\left(x_{\min }^{k}\right) \leq \eta_{1} \text { and } \frac{\left|f_{\min }^{k}-f^{*}\right|}{\max \left\{1,\left|f^{*}\right|\right\}} \leq \eta_{2}
$$

are satisfied, for sufficiently small tolerances $\eta_{1}, \eta_{2}>0$, where $x_{\min }^{k}$ is the best computed solution to the problem, i.e., is the feasible center point of the hyper- 
rectangle that has the least function value $f_{\min }^{k}$. However, if conditions (9) are not satisfied, the algorithm runs until a maximum number of function evaluations, $N f e_{\text {max }}$, is reached.

\section{Numerical Experiments}

In these preliminary numerical experiments, a set of seven benchmark problems with $n \leq 5$ is used. The MATLAB ${ }^{\circledR}$ (MATLAB is a registered trademark of the MathWorks, Inc.) programming language is used to code the algorithm and the tested problems. The parameter values for the algorithm are set as follows: $\epsilon=$ $1 \mathrm{E}-04, G_{\max }=10, L_{\max }=10, \varepsilon=1 \mathrm{E}-06, \eta_{1}=1 \mathrm{E}-04, \eta_{2}=1 \mathrm{E}-04$ and $N f e_{\max }=$ $1 \mathrm{E}+05$. Due to the random issue present in the algorithm, namely the selection of the candidate hyperrectangles, each problem is run five times. The reported results in the subsequent tables correspond to average values obtained in the five runs.

First, we consider two problems, one has 2 variables and 2 inequality constraints and the other 3 variables and 3 equality constraints, to show the effectiveness of the proposed strategy based on the penalty auxiliary function (6) when compared to the more usual L1 penalty-based technique.

Problem 1. (Problem 8 in [19])

$$
\begin{aligned}
& \min _{x \in \Omega} x_{1}^{4}-14 x_{1}^{2}+24 x_{1}-x_{2}^{2} \\
& \text { s. t. } x_{2}-x_{1}^{2}-2 x_{1} \leq-2 \\
& \quad-x_{1}+x_{2} \leq 8
\end{aligned}
$$

with $\Omega=\left\{x \in \mathbb{R}^{2}:-8 \leq x_{1} \leq 10,0 \leq x_{2} \leq 10\right\}$ and $f^{*}=-118.70$.

Problem 2. (Problem 5 in [19])

$$
\begin{aligned}
& \min _{x \in \Omega} x_{3} \\
& \text { s. t. } 30 x_{1}-6 x_{1}^{2}-x_{3}=-250 \\
& \\
& 20 x_{2}-12 x_{2}^{2}-x_{3}=-300 \\
& \\
& 0.5\left(x_{1}+x_{2}\right)^{2}-x_{3}=-150
\end{aligned}
$$

with $\Omega=\left\{x \in \mathbb{R}^{3}: 0 \leq x_{1} \leq 9.422,0 \leq x_{2} \leq 5.903,0 \leq x_{3} \leq 267.42\right\}$ and $f^{*}=201.16$.

To analyze the gain in efficiency of Algorithm 2, we report in Table 1 the average values of $f\left(f_{\min }\right), \theta\left(\theta\left(x_{\min }\right)\right)$, number of iterations $(k)$ and number of function evaluations $(\mathrm{Nfe})$ obtained after the 5 runs using the stopping conditions in (9) (or a maximum of $1 \mathrm{E}+05$ function evaluations is reached). The standard deviation of the obtained $f$ values (St.D.) is also reported. From the results in Table 1 we can conclude that the algorithm with the penalty 6 gives significantly better results than with the penalty L1. Similarly, from Table 2 we conclude that penalty (6) performs much better compared to L1. The algorithm 
Table 1. Comparison between penalty functions, when solving Problem 1 .

\begin{tabular}{crccccc}
\hline Algorithm 2 & $\mu$ & $f_{\min }$ & $S t . D$. & $\theta\left(x_{\min }\right)$ & $k$ & $N f e$ \\
\hline penalty (6) & 0.5 & -118.691829 & $2.26 \mathrm{E}-03$ & $0.00 \mathrm{E}+00$ & 109 & 2175 \\
& 1 & -118.692153 & $2.01 \mathrm{E}-03$ & $0.00 \mathrm{E}+00$ & 86 & 1457 \\
& 10 & -118.688994 & $2.65 \mathrm{E}-04$ & $0.00 \mathrm{E}+00$ & 44 & 526 \\
& 100 & -118.688657 & $1.69 \mathrm{E}-04$ & $0.00 \mathrm{E}+00$ & 77 & 1121 \\
L1 penalty & 0.5 & $(-217)$ & $(6.15 \mathrm{E}-05)$ & $(4.5 \mathrm{E}+00)$ & $(4848)$ & $>1 \mathrm{E}+05$ \\
& 1 & $(-217)$ & $(6.15 \mathrm{E}-05)$ & $(4.5 \mathrm{E}+00)$ & $(4852)$ & $>1 \mathrm{E}+05$ \\
& 10 & $(-215)$ & $(5.02 \mathrm{E}-05)$ & $(4.0 \mathrm{E}+00)$ & $(4828)$ & $>1 \mathrm{E}+05$ \\
& 100 & -118.689247 & $6.48 \mathrm{E}-04$ & $0.00 \mathrm{E}+00$ & 72 & 865 \\
\hline
\end{tabular}

In parentheses, achieved values when the algorithm stops due to $N f e>1 \mathrm{E}+05$.

Table 2. Comparison between penalty functions, when solving Problem 2 .

\begin{tabular}{crccccc}
\hline Algorithm 2 & $\mu$ & $f_{\min }$ & St.D. & $\theta\left(x_{\min }\right)$ & $k$ & $N f e$ \\
\hline penalty (6) & 0.5 & 201.159343 & $0.00 \mathrm{E}+00$ & $7.83 \mathrm{E}-05$ & 43 & 577 \\
& 1 & 201.159343 & $0.00 \mathrm{E}+00$ & $7.83 \mathrm{E}-05$ & 43 & 543 \\
& 10 & 201.159343 & $0.00 \mathrm{E}+00$ & $7.83 \mathrm{E}-05$ & 45 & 624 \\
& 100 & 201.159343 & $0.00 \mathrm{E}+00$ & $7.83 \mathrm{E}-05$ & 41 & 531 \\
penalty L1 & 0.5 & $(201)$ & $(0.00 \mathrm{E}+00)$ & $(7.6 \mathrm{E}-04)$ & $(8803)$ & $>1 \mathrm{E}+05$ \\
& 1 & $(201)$ & $(0.00 \mathrm{E}+00)$ & $(1.4 \mathrm{E}-04)$ & $(7612)$ & $>1 \mathrm{E}+05$ \\
& 10 & 201.159343 & $0.00 \mathrm{E}+00$ & $7.83 \mathrm{E}-05$ & 320 & 5864 \\
& 100 & 201.159343 & $0.00 \mathrm{E}+00$ & $7.83 \mathrm{E}-05$ & 45 & 577
\end{tabular}

In parentheses, achieved values when the algorithm stops due to $N f e>1 \mathrm{E}+05$.

with the penalty L1 works better with the larger values of the weighing parameter while the performance of the algorithm with penalty (6) is not too much affected by the value of $\mu$.

In Table 3 we compare the results obtained by Algorithm 2 based on the penalty auxiliary function (6) for two values of the weighting parameter (that provide the best results among the four tested) with those obtained by previous DIRECT-type strategies that rely on the filter methodology to reduce both the constraint violation and objective function 8 8 17. The results are also compared to those obtained by variants DIRECT-GL and DIRECT-GLce reported in 14. We note that the reported $f_{\min }, \theta\left(x_{\min }\right), k$ and $N f e$ selected from [8] correspond also to average values, while the values from the other papers in comparison correspond to just a single solution (one run of deterministic methods). A slight gain in efficiency of the proposed penalty-based heuristic DIRECT algorithm has been detected.

To analyze the performance of the Algorithm 2 when compared to the strategy proposed in [15] and two filter-based DIRECT algorithms (in [817]), we consider the problem Gomez \#3 (available in [15]): 
Table 3. Comparative results for Problems 1 and 2 .

\begin{tabular}{|c|c|c|c|c|c|c|}
\hline & $\mu$ & $f_{\min }$ & $\theta\left(x_{\min }\right)$ & $k$ & $N f e$ & $f^{*}$ \\
\hline \multicolumn{7}{|l|}{ Problem 1 } \\
\hline \multirow{2}{*}{ Algorithm 2(penalty 6) } & 10 & -118.688994 & $0.00 \mathrm{E}+00$ & 44 & 526 & \multirow[t]{8}{*}{-118.70} \\
\hline & 100 & -118.688657 & $0.00 \mathrm{E}+00$ & 77 & 1121 & \\
\hline DIRECT-type $^{a}$ [ & & -118.700976 & $0.00 \mathrm{E}+00$ & 19 & 823 & \\
\hline DIRECT-type $^{b}$ [8] & & -118.700976 & $0.00 \mathrm{E}+00$ & 19 & 797 & \\
\hline DIRECT-type $^{c}$ [8] & & -118.692210 & $0.00 \mathrm{E}+00$ & 23 & 689 & \\
\hline filter-based DIRECT [17] & & -118.700976 & $0.00 \mathrm{E}+00$ & 23 & 881 & \\
\hline DIRECT-GLc in [14] & & -118.6892 & - & - & 1197 & \\
\hline DIRECT-GLce in [14] & & -118.6898 & - & - & 1947 & \\
\hline \multicolumn{7}{|l|}{ Problem 2} \\
\hline \multirow[t]{2}{*}{ Algorithm 2 (penalty 6) } & 1 & 201.159343 & $7.83 \mathrm{E}-05$ & 43 & 543 & \multirow[t]{8}{*}{201.16} \\
\hline & \multirow[t]{7}{*}{100} & 201.159343 & $7.83 \mathrm{E}-05$ & 41 & 531 & \\
\hline DIRECT-type ${ }^{a}$ 8 & & 201.159343 & $7.83 \mathrm{E}-05$ & 30 & 1015 & \\
\hline DIRECT-type $^{b}$ [8] & & 201.159343 & $7.83 \mathrm{E}-05$ & 30 & 883 & \\
\hline DIRECT-type $^{c}$ [8] & & 201.159343 & $7.83 \mathrm{E}-05$ & 30 & 769 & \\
\hline filter-based DIRECT [17] & & 201.159343 & $7.83 \mathrm{E}-05$ & 30 & 1009 & \\
\hline DIRECT-GLc in 14 & & 201.1593 & - & - & 819 & \\
\hline DIRECT-GLce in [14] & & 201.1593 & - & - & 819 & \\
\hline \multicolumn{7}{|c|}{$\begin{array}{l}{ }^{a}{ }^{a} \text { with filter; }{ }^{b} \text { with filter and upper bounds on } f \text { and } \theta \text {; } \\
{ }^{c} \text { with filter and upper bounds on } f \text { and } \theta \text { as well as a heuristic. }\end{array}$} \\
\hline
\end{tabular}

Problem 3.

$$
\begin{aligned}
& \min _{x \in \Omega}\left(4-2.1 x_{1}^{2}+\frac{x_{1}^{4}}{3}\right) x_{1}^{2}+x_{1} x_{2}+\left(-4+4 x_{2}^{2}\right) x_{2}^{2} \\
& \text { s. t. }-\sin \left(4 \pi x_{1}\right)+2 \sin ^{2}\left(2 \pi x_{2}\right) \leq 0
\end{aligned}
$$

with $\Omega=\left\{x \in \mathbb{R}^{2}:-1 \leq x_{i} \leq 1, i=1,2\right\}$.

Table 4 compares the performance of the tested algorithms. Our Algorithm2 was tested with four different values of the fixed weighting parameter. When solving the Problem 3, our algorithm reports a considerable sensitivity to the selected $\mu$ value, with a better performance achieved when small values are used.

The following problem, known as T1 is tested with 3 different values of $n$.

Problem 4 .

$$
\begin{aligned}
& \min _{x \in \Omega} \sum_{i=1}^{n} x_{i} \\
& \text { s. t. } \sum_{i=1}^{n} x_{i}^{2} \leq 6
\end{aligned}
$$

with $\Omega=\left\{x \in \mathbb{R}^{n}:-1 \leq x_{i} \leq 1, i=1, \ldots, n\right\}$.

The results obtained by Algorithm 2, and those in [14] (variants DIRECTGLc and DIRECT-GLce), as well as the results obtained by the variant DIRECTGL and the original DIRECT (when they are implemented in a penalty-based 
Table 4. Comparison results when solving Problem 3

\begin{tabular}{lrccccc}
\hline & $\mu$ & $f_{\text {min }}$ & $\theta\left(x_{\text {min }}\right)$ & $k$ & $N f e$ & $f^{*}$ \\
\hline Algorithm 2](penalty [6]) & 0.5 & -0.971021 & $2.45 \mathrm{E}-05$ & 51 & 606 & -0.9711 \\
& 1 & -0.971018 & $1.34 \mathrm{E}-05$ & 74 & 983 & \\
& 10 & -0.971018 & $1.34 \mathrm{E}-05$ & 455 & 12952 & \\
& 100 & $(-0.97)$ & $(4.4 \mathrm{E}-05)$ & $(2625)$ & $>1 \mathrm{E}+05$ & \\
DIRECT-type $^{a}$ [8] & & -0.971006 & $6.00 \mathrm{E}-05$ & 17 & 615 & \\
DIRECT-type $^{b}$ [8] & & -0.971006 & $6.00 \mathrm{E}-05$ & 17 & 683 & \\
DIRECT-type $^{c}$ [8] & & -0.971041 & $3.17 \mathrm{E}-05$ & 18 & 555 & \\
filter-based DIRECT [17] & & - & - & -18 & 733 & \\
DIRECT in [15] & & - & - & - & 513 & \\
\hline
\end{tabular}

In parentheses, the achieved values when the algorithm stops due to $N f e>1 \mathrm{E}+05$.

${ }^{a}$ with filter; ${ }^{b}$ with filter and upper bounds on $f$ and $\theta$;

${ }^{c}$ with filter and upper bounds on $f$ and $\theta$ as well as a heuristic.

"_" information not available.

strategy with penalty function L1) are shown in Table 5 for comparison. Our algorithm with the penalty (6) works much better with the smaller values of the fixed weighting parameter and those results outperform in general the other results in comparison, for the same solution quality accuracy, as far as function evaluations are concerned.

Finally, the last 3 problems, known as g04, g06 and g08 in [16], have inequality constraints.

\section{Problem 5.}

$$
\begin{aligned}
& \min _{x \in \Omega} 5.3578547 x_{3}^{2}+0.8356891 x_{1} x_{5}+37.293239 x_{1}-40792.141 \\
& \text { s. t. } 0 \leq 85.334407+0.0056858 x_{2} x_{5}+0.0006262 x_{1} x_{4}-0.0022053 x_{3} x_{5} \leq 92 \\
& 90 \leq 80.51249+0.0071317 x_{2} x_{5}+0.0029955 x_{1} x_{2}+0.0021813 x_{3}^{2} \leq 110 \\
& 20 \leq 9.300961+0.0047026 x_{3} x_{5}+0.0012547 x_{1} x_{3}+0.0019085 x_{3} x_{4} \leq 25
\end{aligned}
$$

with $\Omega=\left\{x \in \mathbb{R}^{5}: 78 \leq x_{1} \leq 102,33 \leq x_{2} \leq 45,27 \leq x_{i} \leq 45, i=3,4,5\right\}$.

Problem 6 .

$$
\begin{aligned}
& \min _{x \in \Omega}\left(x_{1}-10\right)^{3}+\left(x_{2}-20\right)^{3} \\
& \text { s. t. }-\left(x_{1}-5\right)^{2}-\left(x_{2}-5\right)^{2} \leq-100 \\
& \quad\left(x_{1}-6\right)^{2}+\left(x_{2}-5\right)^{2} \leq 82.81
\end{aligned}
$$

with $\Omega=\left\{x \in \mathbb{R}^{2}: 13 \leq x_{1} \leq 100,0 \leq x_{2} \leq 100\right\}$.

Problem 7 .

$$
\begin{aligned}
\min _{x \in \Omega} & -\frac{\sin ^{3}\left(2 \pi x_{1}\right) \sin \left(2 \pi x_{2}\right)}{x_{1}^{3}\left(x_{1}+x_{2}\right)} \\
\text { s. t. } & x_{1}^{2}-x_{2}+1 \leq 0 \\
& 1-x_{1}-\left(x_{2}-4\right)^{2} \leq 0
\end{aligned}
$$

with $\Omega=\left\{x \in \mathbb{R}^{2}: 0 \leq x_{i} \leq 10, i=1,2\right\}$. 
Table 5. Comparison results when solving Problem 4

\begin{tabular}{|c|c|c|c|c|c|c|}
\hline & $\mu$ & $f_{\min }$ & $\theta\left(x_{\min }\right)$ & $k$ & Nfe & $f^{*}$ \\
\hline \multicolumn{7}{|l|}{$n=2$} \\
\hline \multirow{4}{*}{ Algorithm 2(penalty 6) } & 0.5 & -3.464079 & $3.60 \mathrm{E}-05$ & 26 & 383 & -3.4641 \\
\hline & 1 & -3.464052 & $3.30 \mathrm{E}-05$ & 26 & 370 & \\
\hline & 10 & -3.464106 & $9.29 \mathrm{E}-05$ & 40 & 723 & \\
\hline & 100 & -3.464106 & $7.68 \mathrm{E}-05$ & 85 & 3927 & \\
\hline DIRECT-type $^{a}$ [8] & & -3.464106 & $9.29 \mathrm{E}-05$ & 14 & 1395 & \\
\hline DIRECT-type $^{b}$ [8] & & -3.464106 & $9.29 \mathrm{E}-05$ & 14 & 893 & \\
\hline DIRECT-type $^{c}$ [8] & & -3.464106 & $5.72 \mathrm{E}-05$ & 13 & 335 & \\
\hline \multirow{2}{*}{ DIRECT-L1 in [14] } & 10 & - & - & - & 3345 & \\
\hline & 100 & - & - & - & 8229 & \\
\hline \multirow{2}{*}{ DIRECT-GL-L1 in 14 } & 10 & - & - & - & 1221 & \\
\hline & 100 & - & - & - & 1921 & \\
\hline DIRECT-GLc in [14] & & - & - & - & 1373 & \\
\hline DIRECT-GLce in [14] & & - & - & - & 2933 & \\
\hline \multicolumn{7}{|l|}{$n=3$} \\
\hline \multirow[t]{4}{*}{ Algorithm 2(penalty 6) } & 0.5 & -4.242687 & $7.25 \mathrm{E}-05$ & 266 & 29187 & -4.2426 \\
\hline & 1 & -4.242443 & $4.38 \mathrm{E}-05$ & 104 & 6989 & \\
\hline & 10 & -4.242443 & $0.00 \mathrm{E}+00$ & 110 & 8577 & \\
\hline & $100^{d}$ & -4.242443 & $2.30 \mathrm{E}-05$ & 260 & 85472 & \\
\hline DIRECT-type $^{a}$ 8 & & -4.242443 & $0.00 \mathrm{E}+00$ & 28 & 16885 & \\
\hline DIRECT-type ${ }^{b}$ [8] & & -4.242443 & $0.00 \mathrm{E}+00$ & 35 & 37977 & \\
\hline DIRECT-type $^{c}$ [8] & & -4.242443 & $9.17 \mathrm{E}-05$ & 29 & 3233 & \\
\hline \multirow[t]{2}{*}{ DIRECT-L1 in [14] } & 10 & - & - & - & 66137 & \\
\hline & 100 & - & - & - & $>1 \mathrm{E}+05$ & \\
\hline \multirow[t]{2}{*}{ DIRECT-GL-L1 in [14] } & 10 & - & - & - & 75105 & \\
\hline & 100 & - & - & - & 16625 & \\
\hline DIRECT-GLc in [14] & & - & - & - & 26643 & \\
\hline DIRECT-GLce in [14] & & - & - & - & 8297 & \\
\hline \multicolumn{7}{|l|}{$n=4$} \\
\hline \multirow[t]{4}{*}{ Algorithm 2(penalty (6)) } & 0.5 & -4.898440 & $0.00 \mathrm{E}+00$ & 74 & 9514 & -4.899 \\
\hline & 1 & -4.898440 & $0.00 \mathrm{E}+00$ & 62 & 6201 & \\
\hline & $10^{e}$ & -4.898440 & $3.42 \mathrm{E}-05$ & 133 & 54981 & \\
\hline & $100^{d}$ & -4.898440 & $5.80 \mathrm{E}-05$ & 98 & 31440 & \\
\hline DIRECT-type $^{a}$ 8 & & -4.898847 & $0.00 \mathrm{E}+00$ & 42 & 151753 & \\
\hline DIRECT-type ${ }^{b} 8$ & & -4.898847 & $3.42 \mathrm{E}-05$ & 39 & 78859 & \\
\hline DIRECT-type $^{c}$ [8] & & -4.898440 & $3.30 \mathrm{E}-05$ & 51 & 36219 & \\
\hline \multirow[t]{2}{*}{ DIRECT-L1 in 14] } & 10 & - & - & - & 127087 & \\
\hline & 100 & - & - & - & $>1 \mathrm{E}+05$ & \\
\hline \multirow[t]{2}{*}{ DIRECT-GL-L1 in 14 } & 10 & - & - & - & 180383 & \\
\hline & 100 & - & - & - & 189595 & \\
\hline DIRECT-GLc in 14] & & - & - & - & 192951 & \\
\hline DIRECT-GLce in [14] & & - & - & - & 47431 & \\
\hline \multicolumn{7}{|c|}{$\begin{array}{l}\text { with filter; }{ }^{b} \text { with filter and upper bounds on } f \text { and } \theta \text {; } \\
c \text { with filter and upper bounds on } f \text { and } \theta \text { as well as a heuristic. } \\
d \quad 80 \% \text { successful runs; }{ }^{e} 60 \% \text { successful runs. } \\
\text { "-" information not available. }\end{array}$} \\
\hline
\end{tabular}


Table 6. Comparison results when solving Problems 5,6 and 7.

\begin{tabular}{|c|c|c|c|c|c|c|}
\hline & $\mu$ & $f_{\min }$ & $\theta\left(x_{\min }\right)$ & $k$ & $N f e$ & $f^{*}$ \\
\hline \multicolumn{7}{|l|}{ Problem $5^{a}$} \\
\hline \multirow{5}{*}{ Algorithm 2 (penalty (6)) } & $0.5^{b}$ & -30665.2339 & $9.99 \mathrm{E}-05$ & 387 & 36277 & -30665.53867 \\
\hline & $1^{c}$ & -30665.4237 & $9.99 \mathrm{E}-05$ & 377 & 40331 & \\
\hline & 10 & -30665.2450 & $9.96 \mathrm{E}-05$ & 132 & 5119 & \\
\hline & 100 & -30665.2329 & $9.79 \mathrm{E}-05$ & 133 & 5247 & \\
\hline & 1000 & -30665.2329 & $9.79 \mathrm{E}-05$ & 135 & 5746 & \\
\hline DIRECT-GL-L1 in 14 & 1000 & - & - & - & 1799 & \\
\hline DIRECT-GLc in [14] & & - & - & - & 5907 & \\
\hline DIRECT-GLce in 14. & & -30663.5708 & - & - & 21355 & \\
\hline eDIRECT-C in [16] & & -30665.5385 & - & - & 65 & \\
\hline \multicolumn{7}{|l|}{ Problem $6^{a}$} \\
\hline \multirow[t]{5}{*}{ Algorithm 2 (penalty 60$)$} & 0.5 & -6961.9092 & $6.86 \mathrm{E}-05$ & 120 & 2815 & -6961.81387558 \\
\hline & 1 & -6961.8763 & $4.80 \mathrm{E}-05$ & 118 & 2699 & \\
\hline & 10 & -6961.8088 & $2.18 \mathrm{E}-05$ & 114 & 2758 & \\
\hline & 100 & -6961.7868 & $1.66 \mathrm{E}-05$ & 121 & 2939 & \\
\hline & 1000 & -6961.8150 & $2.77 \mathrm{E}-05$ & 186 & 4941 & \\
\hline DIRECT-GL-L1 in 14 & $1000^{d}$ & - & - & - & 289 & \\
\hline DIRECT-GLc in 14] & & - & - & - & 3461 & \\
\hline DIRECT-GLce in 14 & & -6961.1798 & - & - & 6017 & \\
\hline eDIRECT-C in 16 & & -6961.8137 & - & - & 35 & \\
\hline \multicolumn{7}{|l|}{ Problem 7} \\
\hline \multirow{5}{*}{ Algorithm 2 (penalty (6) } & 0.5 & -0.095825 & $0.00 \mathrm{E}+00$ & 18 & 174 & -0.095825 \\
\hline & 1 & -0.095825 & $0.00 \mathrm{E}+00$ & 16 & 158 & \\
\hline & 10 & -0.095825 & $0.00 \mathrm{E}+00$ & 16 & 152 & \\
\hline & 100 & -0.095825 & $0.00 \mathrm{E}+00$ & 16 & 164 & \\
\hline & 1000 & -0.095825 & $0.00 \mathrm{E}+00$ & 15 & 153 & \\
\hline DIRECT-GL-L1 in 14 & 1000 & - & - & - & 471 & \\
\hline DIRECT-GLc in 14] & & - & - & - & 471 & \\
\hline DIRECT-GLce in 14 & & -0.0958 & - & - & 1507 & \\
\hline eDIRECT-C in [16] & & -0.0958 & - & - & 154 & \\
\hline $\begin{array}{l}{ }^{a} \text { results for } \eta_{2}=1 \mathrm{E}-05 ;{ }^{b}{ }^{c} 80 \% \text { successful runs; }{ }^{d} \mathrm{fi} \\
{ }^{b} \text { "_ information not availa }\end{array}$ & $\begin{array}{l}0 \% \text { suc } \\
\text { l solut }\end{array}$ & $\begin{array}{l}\text { ccessful runs; } \\
\text { ion outside t }\end{array}$ & feasibl & . & & \\
\hline
\end{tabular}

The results obtained by our Algorithm 2 for five values of $\mu$ are compared to those of the variants DIRECT-GL-L1, DIRECT-GLc and DIRECT-GLce in 14, and eDIRECT-C from [16. We remark that eDIRECT-C incorporates a local minimization search (MATLAB fmincon). From the results in Table 6 we may conclude that contrary to Problem 5 which presents significantly better results with the larger fixed $\mu$ values, the other two problems report reasonable good 
performances with all the tested $\mu$ values, competing with the other algorithms is comparison.

\section{Conclusions}

In this paper, we present an extension of the heuristic DIRECT method (available in [8) to solve nonlinear CGO problems. The herein proposed extension transforms the CGO problem (1) into a BCGO one, using a penalty strategy based on the penalty auxiliary function (6). We have analyzed the performance of the penalty-based heuristic DIRECT algorithm for a set of fixed penalty parameter values, using well-known benchmark CGO problems. Neither too small nor too large parameter values $(1,10$ and 100) have produced results that show the robustness and efficiency of proposed algorithm hereby competing favourably with other available DIRECT-type algorithms.

Although, for now, we have considered a fixed value for the parameter $\varepsilon$ (in the definition of the penalty (6p), we feel that a sequence of decreased values may further improve the efficiency of the algorithm. This will be an issue for future research.

Acknowledgments. The authors wish to thank two anonymous referees for their comments and suggestions to improve the paper.

This work has been supported by FCT - Fundação para a Ciência e Tecnologia within the R\&D Units Project Scope: UIDB/00319/2020, UIDB/00013/2020 and UIDP/00013/2020 of CMAT-UM.

\section{References}

1. Jones D.R., Perttunen C.D., Stuckman B.E.: Lipschitzian optimization without the Lipschitz constant. based on exact penalty functions. J. Optim. Theory Appl. 79(1), 157-181 (1993)

2. Hendrix, E.M.T., G.-Tóth, B.: Introduction to Nonlinear and Global Optimization. Optimization and its Applications 37, Springer-Verlag (2010)

3. Sergeyev, Y.D.D., Kvasov, D.E.: Deterministic Global Optimization: An Introduction to the Diagonal Approach. SpringerBriefs in Optimization Series, SpringerVerlag (2017)

4. Gablonsky J.M., Kelley C.T.: A locally-biased form of the DIRECT algorithm. J. Glob. Optim. 21(1), 27-37 (2001)

5. Di Pillo, G., Liuzzi, G., Lucidi, S., Piccialli, V., Rinaldi, F.: A DIRECT-type approach for derivative-free constrained global optimization. Comput. Optim. Appl. 65(2), 361-397 (2016)

6. Paulavičius, R., Sergeyev, Y.D., Kvasov, D.E., Žilinskas, J.: Globally-biased DISIMPL algorithm for expensive global optimization. J. Glob. Optim. 59(2-3), 545567 (2014)

7. Stripinis, L., Paulavičius, R., Žilinskas, J.: Improved scheme for selection of potentially optimal hyper-rectangles in DIRECT. Optim. Lett. 12(7), 1699-1712 (2018) 
8. Costa, M.F.P., Fernandes, E.M.G.P., Rocha, A.M.A.C.: A Two-phase heuristic coupled DIRECT method for bound constrained global optimization. EUROGEN 2019 Conference Proceedings, 8 pages, Guimarães, Portugal

9. Paulavičius, R., Žilinskas, J.: Simplicial Lipschitz optimization without the Lipschitz constant. J. Glob. Optim. 59, 23-40 (2014)

10. Liu, H., Xu, S., Wang, X., Wu, J., Song, Y.: A global optimization algorithm for simulation-based problems via the extended DIRECT scheme. Eng. Optim. 47(11), 1441-1458 (2015)

11. Paulavičius, R., Sergeyev, Y.D., Kvasov, D.E., Žilinskas, J.: Globally-biased BIRECT algorithm with local accelerators for expensive global optimization. Expert. Syst. Appl. 144, 113052 (2020)

12. Di Pillo, G., Lucidi, S., Rinaldi, F.: An approach to constrained global optimization based on exact penalty functions. J. Glob. Optim. 54(2), 251-260 (2012)

13. Di Pillo, G., Lucidi, S., Rinaldi, F.: A derivative-free algorithm for constrained global optimization based on exact penalty functions. J. Optim. Theory Appl. 164, 862-882 (2015)

14. Stripinis, L., Paulavičius, R., Žilinskas, J.: Penalty functions and two-step selection procedure based DIRECT-type algorithm for constrained global optimization. Struct. Multidisc. Optim. 59, 2155-2175 (2019)

15. Jones D.R.: The DIRECT global optimization algorithm. In: Floudas, C., Pardalos, P. (Eds.) Encyclopedia of Optimization, pp. 431-440. Kluwer Academic Publisher, Boston (2001)

16. Liu H., Xu, S., Chen X., Wang, X., Ma, Q.: Constrained global optimization via a DIRECT-type constraint-handling technique and an adaptive metamodeling strategy. Struct. Multidisc. Optim. 55(1), 155-177 (2017)

17. Costa, M.F.P., Rocha, A.M.A.C., Fernandes, E.M.G.P.: Filter-based DIRECT method for constrained global optimization. J. Glob. Optim. 71(3), 517-536 (2018)

18. Gablonsky J.M.: DIRECT version 2.0 user guide. Technical Report CRSC-TR-0108, Center for Research in Scientific Computation, North Carolina State University (2001)

19. Birgin, E.G., Floudas, C.A., Martínez, J.M.: Global minimization using an Augmented Lagrangian method with variable lower-level constraints. Technical Report MCDO121206, Department of Computer Science IME-USP, University of São Paulo (2007) http://www.ime.usp.br/ egbirgin/ 